\title{
Experiencia docente en procesos de mejora de la eficacia escolar: la Ley de Subvención Escolar Preferencial en un establecimiento municipalizado de la comuna de La Florida
}

\author{
Manuel Pineda Torres ${ }^{1}$
}

Fecha de recepción: 2 de octubre de 2015

Fecha de aprobación: 23 de marzo de 2016

\begin{abstract}
Resumen
El presente artículo da a conocer las principales condiciones bajo las cuales se desarrollan los planes de mejoramiento escolar adscritos a la Ley de Subvención Escolar Preferencial al interior de un establecimiento municipalizado, reconociendo tales aspectos a partir de la experiencia de los docentes y miembros del equipo de gestión que han sido partícipes de dichos procesos de cambio en los años 2013-2014. La investigación se efectuó en un centro escolar municipal ubicado en la comuna de La Florida, Región Metropolitana de Chile. Para reconocer las condiciones que permean la implementación efectiva de los planes de mejora se consideró pertinente utilizar un diseño cualitativo con un enfoque fenomenológico.

Los hallazgos obtenidos permitieron indicar que, para la implementación de planes de mejoramiento educativo, es necesaria la existencia tanto de condiciones organizacionales óptimas como también la existencia de buenas relaciones entre los miembros de la comunidad educativa. Además, adquiere relevancia el tipo de relaciones que posee el establecimiento con las organizaciones externas que apoyan y supervisan los procesos de cambio, ya que dichas entidades influyen en el funcionamiento de los planes de mejoramiento escolar.
\end{abstract}

Palabras clave: mejora de la eficacia escolar, experiencia docente, organización escolar, condiciones organizacionales.

Sociólogo, Universidad Católica Silva Henríquez. Estudiante del Magíster en Ciencias Sociales, mención Sociología de la Modernización, Universidad de Chile. Contacto: m.pineda.torres@live.com 


\title{
The experience of teaching in processes improving school effectiveness: Preferential School Subsidy Act in a public school of $\mathrm{La}$ Florida municipality
}

\begin{abstract}
This article shows the main conditions under which the school improvement plans are developed, attached to the Preferential School Subsidy Act within a public school. It acknowledges these aspects from the experience of teachers and management staff involved in these processes of change in the years 2013-2014. The research was carried out in a public school located in the municipality of La Florida, Metropolitan Region, Chile. In order to recognize the conditions that pervade the effective implementation of improvement plans, the use of a qualitative design with a phenomenological approach was considered appropriate.

Findings indicate to the need of optimal organizational conditions as well as good relations among members of the educational community for the implementation of plans for educational improvement. Furthermore, the type of relationship among the school and external organizations supporting and monitoring the process of change is becoming increasingly relevant, as these entities affect how school improvement plans work.
\end{abstract}

Keywords: improving school effectiveness, teaching experience, school organization, organization conditions.

\section{Experiência docente em processos de melhora da eficácia escolar: a Lei de Subvenção Escolar Preferencial num estabelecimento municipalizado do município Da Florida}

\footnotetext{
Resumo

O presente artigo descreve as principais condições onde se desenvolvem os planos de melhora escolar associadas à Lei de Subvenção Escolar Preferencial ao interior de um estabelecimento municipalizado, reconhecendo esses aspectos a partir da experiência dos professores e membros da equipe de gestão que foram partícipes destes processos de mudança nos anos 2013-2014. A pesquisa foi realizada numa escola municipal localizada no município de La Florida, Região Metropolitana de Chile. Para reconhecer as condições que permeiam a aplicação efetiva dos planos de melhoria foi considerado pertinente usar um desenho qualitativo com uma abordagem fenomenológica.

Os resultados obtidos permitiram indicar que, para a execução de planos de melhoramento educativo, foi necessária a existência de condições organizacionais óptimas, como também a existência de bons relacionamentos entre os membros da comunidades educativa. Além disso, torna-se relevante o tipo de relações que possui o estabelecimento com as organizações externas
} 
que apoiam e acompanham os processos de mudança, já que estas entidades afetam o funcionamento dos planos de melhora escolar.

Palavras-chave: melhora da eficácia escolar, experiência docente, organização escolar, condições organizacionais.

\section{Introducción}

La implementación de los proyectos de mejora impulsados por la Ley de Subvención Escolar Preferencial -en adelante Ley SEP- a partir del año 2008, se constituyen en procesos de mejoramiento de los establecimientos educacionales en Chile, estimulados por presiones externas para la mejora de la escuela y sus resultados en mediciones académicas nacionales. La Ley SEP busca la consecución de mejoras significativas en la calidad y equidad de la educación, mediante la prestación de recursos económicos a los establecimientos, los cuales posibiliten la entrega de un mejor servicio educacional (MINEDUC, 2008).

La mejora de las escuelas y sus resultados académicos se relaciona con la construcción de sociedades más equitativas y justas. Tedesco (2012) asigna a las escuelas un papel importante en la construcción de sociedades más igualitarias en lo económico, lo cultural y lo social. De tal modo, la realización de mejoras al interior de estas escuelas resulta fundamental para llevar a cabo aquellas aspiraciones relacionadas a la búsqueda de sociedades más igualitarias.

Pese a la implementación de proyectos de mejora, los resultados no han sido los esperados por las autoridades, debido a la mantención de la brecha en el rendimiento de los alumnos, dependiendo del establecimiento en el que realizan sus estudios. Los resultados del Sistema de Medición de Calidad de la Educación (SIMCE) muestran una amplia brecha en cuanto a los rendimientos del alumnado acordes a la dependencia de su establecimiento. Tales contrastes en el rendimiento escolar se replican tanto en las pruebas estandarizadas nacionales -por ejemplo, en la Prueba de Selección Universitaria (PSU), SIMCE-, como también en aquellas mediciones internacionales efectuadas mediante 
las pruebas Trends in International Mathematics and Science Study (TIMSS) y Programme for International Student Assessment (PISA) (Laso, Albarrán y Carrasco, 2012).

Por dichos motivos, es posible observar una problemática importante, ya que no han existido mejoras significativas en los resultados nacionales, pese a la implementación de planes de mejoramiento asociados a la Ley $\mathrm{SEP}^{2}$. Lo anterior permite cuestionar y reflexionar sobre aristas tales como, si se cuenta efectivamente con las condiciones y el apoyo necesario para la concreción de proyectos o procesos de mejora, o bien, si las escuelas y sus funcionarios poseen la capacidad y el compromiso de realizar y mantener mejoras escolares que repercutan tanto en los resultados académicos del sistema escolar, como, a su vez, en la construcción de sociedades más equitativas en lo económico, en lo cultural y en lo social.

A partir de lo anterior, la presente investigación buscó comprender la experiencia de los docentes y, a su vez, conocer las condicionantes organizacionales que limitan -o bien posibilitan- la implementación de planes de mejoramiento escolar en un centro municipalizado de la comuna de La Florida que imparte educación media y básica. El liceo municipal seleccionado es beneficiario de la Ley SEP desde 2013, y además se ha caracterizado por carecer de criterios de selección de sus estudiantes -poseía en 2013 un total de 735 estudiantes, siendo importante señalar que, acorde con los registros de COMUDEF (2013), un 74\% de estos eran vulnerables socioeconómicamente, mientras que un $50 \%$ era posible tipificarlos como prioritarios, por los bajos resultados obtenidos, de manera constante, en mediciones nacionales tales como el SIMCE y PSU.

Desde la teoría, se aprecia el aporte ofrecido por los movimientos teórico-prácticos de la mejora de la eficacia escolar, quienes entregan una serie de condiciones para la realización de

2 La Ley SEP fue promulgada el 25 de enero de 2008 (MINEDUC, 2008). 
cambios organizacionales de tipo endógeno en las escuelas, que tengan repercusiones significativas en los resultados académicos de sus estudiantes. Las principales condiciones que requiere un proceso de mejora de eficacia escolar se vinculan con la existencia de planificación colaborativa al interior de los establecimientos, lo cual requiere disponer de espacios de opinión para la elaboración y posterior ejecución de un plan de mejora. Lo anterior supone la presencia de visiones y metas compartidas en la comunidad educativa, lo cual promueve la participación tanto del profesorado como del resto de los integrantes del establecimiento en procesos de cambio escolar (Ainscow, Hopkins, Southworth y West, 2001, citado en Hernández y Santana, 2010; UNICEF, 2004; Murillo y Krichesky, 2012; Paredes, 2004).

En definitiva, este artículo busca aportar en el plano científico y práctico en las disciplinas de educación y sociología. Lo anterior, a partir del análisis de la implementación de proyectos de mejora adscritos a la Ley SEP. De este modo, se entregarán insumos que faciliten el desarrollo de la educación en esta materia, ya que permiten el conocimiento de aquellos elementos que influyen en la consecución de mejoras escolares, posibilitando disponer de información que contribuya a superar aquellas limitaciones que impiden su desarrollo, lo que facilita la ejecución de mejoras más efectivas a futuro.

\section{Materiales y métodos}

La investigación se inscribe en el paradigma interpretativo. Se utilizó además una metodología cualitativa junto a una perspectiva fenomenológica, ya que el objetivo de la investigación fue dar cuenta de las condiciones organizacionales experimentadas por los docentes de un centro escolar municipal durante los procesos de mejoramiento escolar efectuados los años 2013-2014.

El estudio fue realizado en un establecimiento municipal de La Florida, dependiente de la Corporación Municipal de La Florida, en adelante COMUDEF. La elección del establecimiento se 
debió a que, durante 2013, este desarrolló su primer plan de mejoramiento educativo adscrito a la Ley SEP de forma autónoma, mientras que en 2014 implementó su segundo proyecto en relación con la búsqueda de mejoras significativas en sus puntajes $\mathrm{SIMCE}^{3}$. Además, cuenta con una población escolar caracterizada por sus altos niveles de vulnerabilidad socioeconómica y por su condición prioritaria ( $74 \%$ y 50\%, respectivamente).

Con relación a los objetivos de la investigación, se consideró como informantes a docentes que tuviesen una trayectoria laboral de 2 o más años en el Liceo, que cumplieran una jornada laboral de 35 o más horas, además de que desarrollaran sus labores profesionales exclusivamente en el aula en niveles de enseñanza básica y/o media. No obstante, si bien el estudio tenía su interés en el profesorado y su experiencia, también se consideró la participación de miembros del equipo de gestión y directivos del Liceo, ya que tales funcionarios complementan la información entregada por los docentes.

Por último, se recolectó información mediante la utilización de técnicas como entrevistas en profundidad, entrevistas grupales e información documentada. A continuación se entrega el detalle tanto de docentes y miembros del equipo de gestión -directivos que participaron del proceso de producción de información-, como también de los documentos analizados:

- Entrevistas en profundidad: 7 profesores, 7 funcionarios de gestión, dirección.

- Entrevista grupal: 5 docentes.

El establecimiento bajó sus puntajes en las pruebas de Lectura y Matemáticas en la transición de 2013 a 2014, específicamente en octavo básico y segundo medio. En octavo básico, en Lectura, en 2013 se obtuvo un puntaje de 231 ptos., bajando en 2014 a 192 ptos.; mientras que, en Matemáticas, el puntaje durante el mismo periodo se mantiene. En segundo medio, en Lectura, disminuyó de 232 a 216 ptos., en el caso de Matemáticas la disminución fue de 219 a 208 ptos. 
- Información documentada: Plan Anual de Educación Municipal, Ilustre Municipalidad de La Florida. Proyecto Educativo Institucional del Establecimiento.

La cantidad de entrevistas efectuadas, en el caso de las entrevistas en profundidad, fue de una para cada actor; mientras que se realizó solo una entrevista grupal. Para el análisis y sistematización de la información recopilada se implementó el análisis de contenido temático, expuesto por Pérez (2007). Para facilitar la construcción de categorías se empleó el software de análisis cualitativo Atlas ti.

\section{Resultados}

A continuación se presentan los principales hallazgos del estudio, obtenidos mediante la aplicación de entrevistas en profundidad y una entrevista de carácter grupal. La clasificación de la información se realiza de acuerdo con el año de implementación de los planes efectuados por el establecimiento, los elementos que facilitaron y limitaron la implementación de ambos planes y las estrategias que los informantes proponen para desarrollar de forma efectiva futuros procesos de mejora de la eficacia escolar.

\section{Plan de mejoramiento escolar implementado el 2013}

El profesorado manifestó que la construcción del plan de mejoramiento se caracterizó por la existencia de espacios de participación y de tiempos asignados para elaborar acciones destinadas a la mejora de la organización escolar y sus resultados. En contraste, los miembros del equipo de gestión y directivos señalaron una serie de dificultades durante el proceso de configuración del plan, relacionadas con la demora para definir las actividades del plan y la participación parcial de los directivos y coordinadores de la escuela.

Aquellas ideas en común que posee el estamento docente con los miembros de gestión y directivos respecto de la elaboración del 
proceso de mejora, guardan relación con que existió una participación importante de miembros de la comunidad escolar del establecimiento, pero que se careció de los tiempos necesarios para la construcción del plan de mejoramiento escolar, en adelante PME.

En cuanto a la ejecución del PME 2013, el profesorado indicó que, pese a la existencia de espacios de participación, el proceso se vio dificultado debido a la ausencia de miembros del equipo de gestión y directivos, lo cual incidió en que existiese una baja claridad respecto de las tareas a efectuar. A su vez, los miembros del equipo de gestión y directivos expresaron que gran parte de las dificultades ocurridas durante la ejecución del PME se relacionan con la reciente autonomía del Liceo, lo cual se evidenciaría en su inexperiencia para desarrollar procesos de cambio educativo. Además, se hizo mención a la imposición de acciones por parte del sostenedor. $\mathrm{Al}$ respecto, tanto docentes como miembros de los estamentos de gestión y directivos creen que, a pesar de las dificultades, existió una planificación adecuada de actividades, no obstante que gran parte de éstas no se concretaran. Para los funcionarios, la principal problemática tuvo relación con el manejo de los recursos entregados por la Ley SEP.

\section{Plan de mejoramiento escolar implementado el 2014}

Los docentes en general indicaron que la construcción del PME 2014 tuvo mayores dificultades que el elaborado el 2013. Se menciona que en este proceso, no obstante a la existencia de una mejora en las relaciones con los miembros del equipo de gestión y directivos - muchos de los cuales empiezan a desempeñar sus labores en 2014-, prevalecieron problemas relacionados con el liderazgo de la persona que asume entonces el cargo de director. En relación al cambio de director, se generaron dificultades asociadas al desconocimiento de las decisiones tomadas por los estamentos de gestión y dirección.

Tales elementos produjeron que, durante el proceso, existiese una baja participación de la comunidad educativa y una carencia 
de espacios destinados a la definición de actividades. Las condiciones organizacionales mencionadas contribuyeron, a juicio de los docentes, a gestar la presencia de un trabajo por inercia por parte del profesorado en las actividades contempladas en el PME. Lo anterior cobró mayor relevancia debido a la carencia de tiempos para trabajar en el plan.

A diferencia de la visión que poseen los docentes, los miembros del equipo de gestión y directivos poseen una visión más favorable respecto del proceso de elaboración del PME 2014. Enfatizan en que existió participación de la totalidad de los miembros que conforman la comunidad educativa de la escuela, y que se dio una integración efectiva de los nuevos funcionarios que asumieron sus funciones en el estamento de gestión y/o dirección. Sin embargo, pese a la participación de la comunidad en el proceso de construcción, se mencionó que la elaboración del PME y sus actividades fue una tarea exclusivamente realizada por el equipo de gestión y directivos, ya que no se contaba con el tiempo necesario para efectuar un proceso más participativo, que contemplara a todos los estamentos del establecimiento. A partir de lo anterior, es posible dar cuenta de una contradicción, ya que se afirma la participación de la comunidad, mientras que, simultáneamente, se indica que el proceso fue realizado exclusivamente por funcionarios de gestión y dirección.

Al igual que el proceso de construcción, la ejecución del PME tuvo, para los docentes, variadas dificultades que imposibilitaron su concreción efectiva. Se indicaron limitaciones asociadas a las malas relaciones entre el profesorado y los estamentos superiores y el exceso de funcionarios en el equipo de gestión y dirección. Para los docentes, esto incidió en la claridad que ellos tenían respecto de las tareas que debían efectuar en el PME, ya que, al existir distintos individuos coordinando el proceso, recibían instrucciones disímiles.

Además, hubo carencia de espacios de discusión sobre las actividades a implementar, lo que incidió en el desconocimiento 
de las acciones consignadas en el PME por parte del profesorado. La carencia de información respecto del rol que jugaba cada profesor y las acciones que debía realizar, repercutieron en que estos desarrollaran las acciones requeridas por los estamentos de forma apresurada, por inercia y careciendo de información en cuanto a qué finalidades o metas se buscaba alcanzar mediante estas acciones.

Los miembros del equipo de gestión y directivos poseen una visión opuesta respecto de la expresada por los docentes sobre la ejecución del PME 2014. Los funcionarios manifestaron que el proceso de ejecución se caracterizó por la buena relación que hubo con los docentes, destacando además el rol protagónico y el liderazgo ejercido por el nuevo director de la organización escolar.

Al igual que en el proceso de construcción, mencionan que la ejecución del PME fue realizada de forma exclusiva por los funcionarios del equipo de gestión y directivos. Justifican lo anterior argumentando la carencia de tiempo de los docentes, lo cual dificultó la integración de estos actores en dicho proceso. Sin embargo, también se plantean dificultades asociadas a la convocación de actores claves -como educandos y apoderados- para participar de estos procesos.

\section{Visión de informantes sobre condiciones que limitan y facilitan la implementación de PME}

En cuanto a las condiciones que han limitado la implementación de planes de mejoramiento escolar, los docentes se refirieron a problemas que pueden clasificarse de la siguiente manera: dificultades en las relaciones de la comunidad educativa -especialmente con los equipos de gestión y directivos-, problemas de comunicación e inexistencia de metas compartidas, alta rotación de recursos humanos, carencia de espacios y tiempos para discutir aspectos relacionados con la implementación de planes de mejora, sobrecarga laboral, formas de trabajo carentes de compromiso 
respecto de un proceso de cambio, e inexistencia de trabajo colaborativo en la organización.

En el caso del equipo de gestión y directivos, las condiciones que estos conciben como limitadoras de los PME se podrían categorizar de la siguiente forma: desgaste de los funcionarios de la escuela, baja experiencia del establecimiento en procesos de cambio y falta de compromiso de miembros de la comunidad escolar.

A su vez, ambos estamentos coinciden en citar como limitaciones de importancia: problemas en el nivel de liderazgo directivo, relaciones entre docentes y directivos, carencia de tiempos y, principalmente, problemas con el sostenedor del establecimiento, siendo para esta institución la COMUDEF.

Respecto de las condiciones de la escuela que facilitaron la implementación de los PME, los docentes destacaron elementos asociados a las relaciones entre el estamento docente y los estudiantes, la infraestructura y los recursos que recibía el Liceo por concepto de subvención preferencial, la vocación de parte del profesorado por su trabajo y las presiones/exigencias en cuanto al cumplimiento de metas educativas establecidas por la $\mathrm{CO}-$ MUDEF. No obstante que en las limitaciones de los docentes se mencionaba lo referido a problemas con miembros del equipo de gestión y directivos, el profesorado destaca como facilitador la existencia de buenas relaciones con dichos actores durante el plan de mejora implementado el 2013.

El equipo de gestión y los directivos conciben como facilitadores aspectos asociados tanto a las relaciones fluidas al interior del estamento como las sostenidas con la comunidad educativa en general. Además, también han recalcado la existencia de compromiso por parte de los miembros del equipo, debido a la presencia de metas compartidas en cuanto a los procesos de cambio, y destacan la gestión del director del año 2014 para el alcance de aquellas metas orientadas a mejorar el establecimiento y sus resultados académicos. Por último, también se concibieron como facilitadores, las relaciones entre el estamento directivo y 
los docentes, la disposición de los profesores para participar en los procesos de cambio y el compromiso que estos manifestaron en dichas instancias.

\section{Medidas y estrategias para una mejor implementación de planes de mejora escolar}

A partir de la experiencia en planes de mejoramiento, los funcionarios del establecimiento entregaron una serie de recomendaciones, que permitirían implementar a futuro planes de mejoramiento escolar significativos, que tuviesen una mayor incidencia tanto en el funcionamiento de la organización escolar como en el rendimiento de la escuela en procesos de medición de resultados educativos.

Los docentes manifestaron que es necesario trabajar en elementos relacionados con la identificación de la comunidad escolar con la escuela, además de entregar mayor información sobre los planes de mejora a los individuos que conforman la organización. También se mencionó generar una mayor inclusión de la comunidad en los procesos de mejora e incentivar la participación de los individuos en tales planes, lo cual requeriría redefinir el ejercicio del liderazgo directivo que existe en la institución. Por último, los docentes creen necesaria la existencia de una mayor claridad respecto de lo que ellos deben efectuar en los planes de mejora, además de que exista remuneración de las horas, no consignadas en su jornada laboral, dedicadas a estos proyectos.

Las estrategias propuestas por el equipo de gestión y directivos consisten en fomentar la participación de todos los estamentos que componen la escuela -específicamente de los estudiantes- y generar un mayor compromiso en la comunidad educativa en relación al desarrollo de actividades asociadas a los planes de mejora. Dicho sea de paso, se planteó la necesidad de llevar a cabo la toma de decisiones, al interior de la organización, de forma más consensuada y participativa. 


\section{Discusión}

En esta sección se entrega el análisis teórico de los principales hallazgos descritos en el apartado de resultados. Esto permite la comprensión y reflexión sobre los fenómenos acaecidos en la escuela, y vivenciados por docentes, directivos y funcionarios de gestión, a partir de preceptos teóricos tanto organizacionales, educacionales y sociológicos.

A partir de los hallazgos ya señalados se puede indicar que el centro escolar, durante la implementación de los planes de mejoramiento 2013-2014, contó con una serie de condiciones que posibilitaron su funcionamiento (infraestructura, adquisición de recursos, relaciones positivas entre parte del cuerpo docente y otros estamentos, etc.). Pese a ello, a partir de los hallazgos sobre la experiencia tanto de docentes como de miembros del equipo de gestión y directivos en procesos de mejoramiento escolar, se puede observar una serie de condiciones que dificultaron la realización de tales planes en el establecimiento.

Los elementos de carácter organizacional, tanto internos y externos, como aquellos elementos de tipo subjetivo y relacional influyeron en la implementación de planes de mejoramiento 2013-2014, y en la consecución de objetivos asociados a la mejora de las condiciones organizacionales que posibiliten, a futuro, mayores aprendizajes para los estudiantes.

La escuela, como organización, debe entregar a los individuos que la componen una serie de objetivos comunes (Etzioni, 1993). Sin embargo, los docentes del establecimiento carecían y/o desconocían tanto las metas como los objetivos organizacionales asociados a los proyectos de mejora. Si bien existía una serie de lineamientos comunes relacionados con la enseñanza, se observó la ausencia de una orientación común que posibilitara a los funcionarios realizar acciones para el cumplimiento de tales fines. Dicho aspecto se vio reflejado en la falta de un funcionamiento organizacional que facilitara la implementación de planes de mejora. 
Dicha problemática guarda relación con la forma en que participan, especialmente, docentes y funcionarios de gestión y dirección. La falta de objetivos compartidos que orienten el funcionamiento de la organización merma la integración de los sujetos en la organización y, debido a ello, se generan problemas para alcanzar fines institucionales.

Etzioni (1993) relaciona tal dificultad con la carencia de un interés colectivo en cuanto a los objetivos que posee la organización. Mientras Astudillo (1997) indica que el no funcionamiento de la organización, con base en objetivos claros, se debe a problemas en el nivel de subsistema administrativo, ya que los miembros que lo integran -en el caso estudiado, directivos y miembros del equipo de gestión- no realizan una coordinación adecuada de tareas que faciliten el cumplimiento de los fines del establecimiento.

Prosiguiendo con los postulados de Astudillo (1997), la escuela también es concebida como un sistema abierto a las demandas de su entorno; tales exigencias consisten en la mejora de los resultados académicos, solicitada por las entidades gubernamentales de educación en Chile. Bajo esta conceptualización, es posible apreciar la no correspondencia entre metas organizacionales y aquellas de carácter externo exigida por entidades gubernamentales educacionales.

Por ejemplo, el Ministerio de Educación, en adelante MINEDUC, si bien plantea una serie de requisitos estipulados en la Ley SEP, tales aspectos no permearon de forma eficaz los valores y metas de la organización escolar estudiada, ya que no fueron adoptados por ésta. Se debe recordar que en el establecimiento estudiado se carecía de claridad sobre las metas que buscaban los planes de mejoramiento en el establecimiento. Tal situación también es relevante a partir de la interpretación ofrecida por el marco comprensivo para la mejora de la eficacia escolar, desarrollada por Murillo (2009), en cuanto a la correspondencia de metas generales del establecimiento y su coherencia con las metas del 
sistema educativo. Por tanto, en el centro analizado no existió una correspondencia entre las metas educativas generales ideales, las entregadas por la COMUDEF y las metas particulares del establecimiento en cuanto a los planes de mejoramiento.

Las falencias ya señaladas imposibilitaron el correcto funcionamiento de la organización como un sistema, ya que los problemas en los subsistemas de valores-metas y en el plano administrativo impidieron que la escuela tuviera la articulación e imbricación requerida, tanto para el desarrollo de actividades que la organización consignó como para el cumplimiento de los objetivos que la escuela poseía. Lo mencionado cobra relevancia si se considera la premisa de Astudillo (2007), la cual hace hincapié en que para la realización de procesos de cambio planificado en una organización escolar -como es el caso de la implementación de un plan de mejoramiento- se requiere, debido al carácter sistémico de tales transformaciones, el trabajo de todos los subsistemas en función del cambio a implementar.

En síntesis, la falta de objetivos y metas compartidas en la comunidad escolar generó que los procesos de mejora de la eficacia escolar no tuviesen la efectividad necesaria, ya que el desconocimiento de las finalidades de tales planes incidió en el compromiso y participación de los docentes durante su ejecución. Lo anterior se relaciona con la falta de instancias de reflexión, las cuales permiten que los actores se informen sobre los planes de mejoramiento; tal carencia se manifestó en mayor medida en el plan implementado en 2014.

También los problemas en el nivel de dirección y equipo de gestión jugaron un papel clave en la implementación de los planes de mejoramiento. Tales dificultades se relacionan con las interacciones entre profesores y directivos. No obstante, durante el proceso 2013 los entrevistados señalaron que las relaciones entre los docentes y la directora se caracterizaron por ser de tipo democrático, en contradicción con la relación con el directivo que asume el 2014, ya que este fue tipificado como un líder autori- 
tario, tanto por no considerar la opinión del estamento docente, como también por la imposición de acciones. El tipo de liderazgo ejercido por el director en 2014 corresponde a la categoría de líder autocrático, entregada por Kurt Lewin (Krieger, 2001).

En relación con este aspecto, Krieger (2001) indica que un liderazgo democrático es un elemento fundamental para el correcto funcionamiento de procesos de cambio. En los planes de mejoramiento efectuados en el establecimiento se apreció la falta de liderazgo democrático, particularmente durante el proceso 2014, ya que, además de lo señalado en el párrafo anterior, no existió un individuo que fomentara la participación activa de los funcionarios docentes durante los procesos de cambio y, a su vez, promoviera de forma efectiva la inclusión de la comunidad escolar en la toma de decisiones. Por lo tanto, los procesos efectuados y la toma de decisiones se caracterizaron por ser poco inclusivos, lo cual dificulta la realización de transformaciones consensuadas y efectivas al interior de una organización escolar.

Además de los problemas en las relaciones entre docentes y directivos, hubo inconvenientes en las interacciones al interior del estamento docente y de la comunidad educativa en general. Por tanto, se dificultó la constitución de un trabajo colaborativo, tanto en el nivel de estamento docente, como también en el de comunidad, debido a lo cual primaron formas de trabajo individual y/o fragmentario. Lo señalado se pudo observar en la baja participación de la comunidad educativa de los planes de mejoramiento, principalmente durante 2014.

Los docentes carecían de horas no lectivas que posibilitaran su participación, por tanto su inclusión en el desarrollo de planes de mejora se caracterizó por ser apurada, careciendo del tiempo necesario para un trabajo comprometido y reflexivo. La falta de tiempos se puede asociar además a los bajos espacios de reunión que entregaba la dirección y el equipo de gestión a los actores de la comunidad escolar para la construcción y posterior implementación de planes de mejora escolar. 
No obstante, los problemas en la implementación de los planes no deben ser atribuidos solo a las condiciones de los establecimientos beneficiarios, ya que también hubo dificultades externas a estos que mermaron su realización.

En relación a las formas en que se administra el plan de mejoramiento en el establecimiento, la COMUDEF -como sostenedor-se constituyó en un obstáculo para el funcionamiento de los planes de mejoramiento desarrollados por el liceo. Lo señalado quedó de manifiesto en la falta de autonomía del establecimiento, tanto en la gestión de sus planes como en la elección y ejecución de acciones que éste estimaba necesarias para mejorar. El sostenedor se caracterizó por imponer actividades al establecimiento.

Bolívar (2005) y Paredes (2004) plantean que, para la implementación de procesos de mejora, el centro escolar debe poseer un cierto grado de autonomía que le permita la administración de recursos, lo cual posibilite la utilización de tales insumos en acciones que el colegio vea como esenciales para solventar sus necesidades. Por tanto, las instituciones externas que supervisan -y que además juegan un papel importante- procesos de cambio escolar deben mantener una relación de carácter horizontal con las escuelas en dicha situación. Tal tipo de dinámica permite un proceso de acompañamiento, en el cual la entidad respeta las decisiones del establecimiento, considerando las particularidades y necesidades que reporta; es decir, se constituye en un apoyo más que en un obstáculo.

Las dificultades presentes en la organización, tanto externas como internas, imposibilitaron el correcto funcionamiento de los planes de mejoramiento. Los problemas mencionados permiten dar cuenta de las dificultades para conformar una cultura escolar propicia para la generación de transformaciones, lo cual dificultó la realización de cambios significativos. La carencia y los problemas asociados a la generación de una cultura de la mejora se vio reflejada en el incumplimiento de las condiciones ideales entregadas, tanto por los postulados de la mejora de la eficacia escolar (li- 
derazgo, falta de tiempos, relaciones con el sostenedor, etc.), como también por las dificultades presentes en la interrelación de los subsistemas valórico y administrativo que componen la escuela.

Los problemas para institucionalizar una cultura de la mejora también se apreciaron en la divergencia de opiniones en relación con la implementación de los planes de mejoramiento escolar. Las discordancias observadas en la experiencia de los docentes y miembros del equipo de gestión, en cuanto a su percepción del cambio y las formas en que se ha desarrollado al interior del establecimiento, permitieron visualizar el carácter contradictorio de la cultura que imperaba en el centro escolar. Desde el ámbito teórico, Sepúlveda y Murillo (2012) recalcan que la cultura de la mejora juega un rol clave para la integración efectiva y comprometida de los miembros de una comunidad educativa en un proceso de cambio.

No obstante, pese a todas los impedimentos organizacionales que se observaron en el establecimiento, se han desarrollado los planes de mejoramiento escolar. Las prácticas de los funcionarios del establecimiento durante el desarrollo de tales proyectos, si bien se vieron normadas por una serie de reglamentos y requisitos impuestos por la organización escolar, los docentes aprovecharon aquellos espacios de incertidumbre que dejó la organización.

Tal situación puede ser comprendida a partir de la propuesta teórica de Crozier y Friedberg (1990), quienes indican que los individuos no se encuentran totalmente coaccionados por la organización y sus imposiciones, ya que mantienen un cierto grado de libertad en su actuar. Lo anterior permite comprender por qué el accionar de los individuos se distanció, en ciertas circunstancias, de los requisitos de la Ley SEP y de la organización escolar. Por ende, si bien se llevaron a cabo las acciones en pos de objetivos de la organización, sus prácticas no fueron del todo congruentes con el cambio escolar que buscaba el centro educativo y las instituciones gubernamentales de educación. 
Lo señalado se relaciona con el hecho de que las formas de involucramiento y participación en planes de mejoramiento no se encuentran totalmente normadas ni reglamentadas. Dicho espacio, carente de reglamentaciones, se constituyó en un área de incertidumbre organizacional que entregaba mayor libertad de acción a los individuos.

Para dar una mayor comprensión a las prácticas efectuadas por los docentes en los procesos de mejora escolar y sus contribuciones, o impedimentos, en su desarrollo, se recurre al tipo de prácticas en contextos de cambio desarrolladas por PNUD (2009). Teniendo en consideración esta categorización, las prácticas docentes pueden ser tipificadas bajo el rótulo de "adopción resistida" y "acuerdo de mínimos", mientras que la acción tanto de cierta parte de miembros del equipo de gestión, como también de algunos profesores, puede ser categorizada como "improvisación adaptativa".

Los individuos que llevan a cabo prácticas asociadas a la adopción resistida visualizan los procesos de cambio como perjudiciales. Por tanto, existe una resistencia explicita a estas trasformaciones; pese a ello, estos individuos buscan reinterpretar el sentido del cambio y adaptarlo a sus intereses personales. Dicho tipo de prácticas se manifestó en el actuar de los docentes mediante el cumplimiento de las acciones o requisitos necesarios para llevar a cabo planes de mejora en el establecimiento, ya que si bien no compartían los preceptos de tales procesos de cambio, intentaron compatibilizar y adaptar sus aspiraciones personales con estos.

Por ejemplo, los profesores declararon que los planes de mejoramiento no se constituían como un medio totalmente eficaz para mejorar las condiciones del establecimiento. Pese a ello, sus intereses y motivaciones asociados a la enseñanza contribuyeron a que participaran, tanto de instancias contempladas en los planes de mejora como en la ejecución de acciones que no eran concebidas como idóneas para la mejora de las condiciones educacionales del liceo. 
Las prácticas relacionadas al acuerdo de mínimos, según PNUD (2009), se caracterizan por la alta dependencia y la desconfianza entre los individuos. Los modos de hacer las cosas se definen por una involucración mínima, la cual hace que el actor perciba que no se abusa de sus prácticas. La forma en que participó parte importante del profesorado en los planes, de acuerdo con lo mencionado por los informantes, fue "solo por cumplir"; argumentan ellos que esto se visualiza en el bajo compromiso con las acciones y tareas realizadas por funcionarios en el centro escolar. Además, las tipificaciones negativas realizadas por los docentes y directivos del establecimiento -específicamente las de los miembros que conforman el equipo de 2014- reflejan la baja confianza existente en la comunidad escolar. Lo anterior mermó las aspiraciones de cambio al interior del establecimiento, ya que la presencia de una alta desconfianza entre los funcionarios dificultó sus relaciones y la ejecución de procesos contemplados en los planes de mejora.

En síntesis, las prácticas de acuerdo de mínimos y adopción resistida se relacionan con la ejecución de acciones solo por cumplir con lo requerido por la escuela. Merton (2002) expresa que las acciones ritualistas al interior de un contexto determinado se caracterizan por efectuar las tareas necesarias para el cumplimiento de determinados objetivos, pero los funcionarios que efectúan dichas prácticas carecen de interés y compromiso con la finalidad que desea alcanzar la organización.

En relación con la propuesta tanto de Merton (2002) como de PNUD (2009), los docentes declararon que su trabajo durante la implementación de planes de mejoramiento era de carácter rutinario; por ello, las acciones y tareas consignadas en los procesos de cambio carecieron de compromiso, ya que fueron desarrolladas solamente por cumplir y para no recibir sanciones por parte de los miembros del equipo de gestión y dirección del establecimiento. Las prácticas de algunos docentes se constituyeron como una forma de acción ritualista, ya que, si bien el profesorado cumplió con las normas institucionales, se generó un abandono, 
desinterés y/o rechazo de las finalidades y objetivos por parte del profesorado en relación a los procesos de cambio implementados. "Es, en resumen, el modo de adaptación para buscar en forma individual un escape privado de los peligros y frustraciones (...) abandonando esas metas y aferrándose lo más estrechamente posible a las seguras rutinas de las normas institucionales" (Merton, 2002, p. 230).

El cuerpo docente y el equipo de gestión -especialmente el del año 2013- también realizó prácticas asociadas a la improvisación adaptativa (el individuo asume la función de cumplir con aquellas funciones que los demás miembros no efectúan), ya que debido a la poca claridad de roles, la constante carencia y rotación de personal, tuvieron que desempeñar funciones que no se correspondían con sus labores profesionales. Por ejemplo, durante el plan de mejoramiento 2013 existió un problema con las jefas de unidad técnica, debido a lo cual el resto del equipo de gestión, principalmente la encargada de integración, orientación y la directora de ese entonces, asumieron la implementación del plan de mejoramiento. Tal situación también se vio reflejada en los docentes, porque muchos debieron ejecutar acciones que debían ser realizadas por otros colegas y/o miembros de la comunidad escolar.

En síntesis, se puede afirmar que los postulados de Crozier y Friedberg (1990), PNUD (2009) y Merton (2002) poseen expresa relación y permiten comprender el tipo de acciones que tuvo lugar en el establecimiento durante los procesos de cambio. El centro escolar no tuvo la capacidad de normar del todo la forma en que los funcionarios participaron de las transformaciones escolares. Por lo tanto, entregó la posibilidad de que los docentes llevasen a cabo acciones no cooptadas por los requisitos de la organización, lo cual pudo afectar la generación de los cambios que buscaba implementar el establecimiento.

UNICEF (2004) y Sepúlveda y Murillo (2012) indican que la adopción y adhesión de los docentes a los procesos de cambio es 
fundamental para la consecución de mejoras significativas. Pese a ello, tal condición no se dio en el establecimiento estudiado, ya que parte del profesorado se resistió y desarrolló prácticas no congruentes con los cambios que se deseaban implementar mediante planes de mejoramiento escolar.

Si bien en el análisis desarrollado he dado cuenta de una serie de problemas de tipo organizacional interno, externo y sobre las prácticas efectuadas por los funcionarios, resulta importante entregar un análisis de las formas en que se relacionan los docentes con otros individuos durante un proceso de cambio, y como su apreciación e identificación puede ser decisiva en procesos de transformación escolar. Es decir, la importancia de la subjetividad y las relaciones de los funcionarios durante procesos de cambio.

Por lo tanto, teniendo en consideración las prácticas desarrolladas durante los planes de mejoramiento y las condiciones laborales que declararon los docentes, se pudo dar cuenta del contenido subjetivo inherente que permeó las prácticas del profesorado. El contenido subjetivo de tales prácticas se vio reflejado tanto en el interés de los docentes por participar en tales planes, el grado de compromiso que estos poseían con el cambio y sus tareas, como también en las relaciones que establecieron con la comunidad escolar, principalmente con otros funcionarios.

Berger y Luckmann (2003) indican que las tipificaciones realizadas por un individuo durante una interacción son determinantes en las relaciones futuras que sostendrán tales sujetos. Extrapolando este postulado a las tipificaciones negativas de los docentes hacia sus propios pares, miembros del equipo de gestión, dirección (específicamente para el director del año 2014) y sostenedor, es posible comprender la incidencia de las subjetividades individuales en el funcionamiento de planes de mejoramiento. La tipificación realizada por docentes repercutió en las formas en que trató, se relacionó y aprendió a los miembros de la comunidad escolar. 
A su vez, los postulados entregados por Schütz (1993) permiten dar una mayor profundidad analítica a lo indicado por Berger y Luckmann (2003). La corriente de la conciencia interna de los individuos, duree, retiene aquellas experiencias discretas que el docente ha tenido con miembros del establecimiento. Por lo tanto, debido a la primacía de vivencias significativas de carácter negativo experimentadas por los docentes, de acuerdo con los hallazgos mencionados en la sección anterior, se puede entender que incidieron de forma importante en el profesorado, su subjetividad y en sus interrelaciones con la comunidad educativa del centro.

Por lo tanto, a partir de la interrelación de los preceptos teóricos entregados por Schütz (1993) y Berger y Luckmann (2003), es posible develar el motivo por el cual prevalecieron problemas en las relaciones cotidianas y laborales, y que generó la desconfianza de los docentes hacia el resto de la comunidad educativa. Además, tales tipificaciones y vivencias significativas, que son de tipo personal, ante las constantes interacciones de los docentes con sus pares y/o miembros de otros estamentos, generaron la prevalencia de percepciones negativas respecto de otros miembros de la comunidad escolar.

Lo anterior constituyó un ambiente en el que imperó un sentido común caracterizado por la tipificación negativa de los otros y la desconfianza hacia estos. Debido a ello, los aspectos asociados con la experiencia de los docentes, tanto en el establecimiento como durante la implementación de planes de mejoramiento, adquieren relevancia. Aquellas experiencias de tipo discreto y las tipificaciones que tenían los docentes de los miembros de la comunidad permearon el grado de involucración del profesorado.

Prosiguiendo con los axiomas teóricos de Schütz (1993) y Berger y Luckmann (2003), las experiencias discretas y tipificaciones negativas de los docentes también afectaron las posibilidades de cambio que el profesorado creía poder lograr mediante planes de mejoramiento escolar. Por lo tanto, las conductas y/o prácticas 
desfavorables de los docentes durante la realización de cambios, no responden solo a situaciones o elementos emergentes y/o recientes, sino que datan de experiencias discretas y tipificaciones desfavorables, que se asocian a una concepción negativa del funcionamiento de los planes de mejoramiento escolar y de las instituciones que supervisan su desarrollo.

De acuerdo con lo señalado sobre el concepto de experiencias discretas, los profesores, al tener vivencias negativas relacionadas con el desarrollo de planes de mejoramiento como también tipificaciones negativas sobre la administración del sostenedor y las formas en que el establecimiento gestiona la implementación de planes, gestaron un desgaste que repercutió en sus esperanzas de cambio efectivo en el centro escolar. Tal desgaste, expresado tanto por docentes como por parte de los miembros del equipo de gestión y dirección, se erigió como un elemento clave durante el desarrollo de planes de mejora, ya que afectó el grado de compromiso con el que participaron los docentes en los cambios de la organización.

En este sentido, existe una contradicción entre los postulados ya mencionados, las posibilidades de cambio que señalaron los funcionarios y lo indicado tanto por Fundación Chile (2010) como por Raczynski, Muñoz, Weinstein, y Pascual (2013), ya que estos mencionan que gran parte de los docentes y directivos de los establecimientos municipalizados poseen altas expectativas en cuanto a los cambios que pueden generarse mediante los proyectos adscritos a la Ley SEP. En contraste, los funcionarios, especialmente docentes, debido a sus experiencias negativas durante estos planes, ven baja posibilidad de transformación mediante estos. Por ende, se observa el predominio de una desesperanza aprendida en la organización escolar.

No obstante, todas las dificultades expuestas en el presente apartado guardan relación con que los procesos de mejora de la eficacia escolar se caracterizan por las dificultades inherentes que poseen. En este sentido, Murillo y Krichesky (2012) indican que 
los proyectos de mejoramiento en centros escolares no poseen una vía de carácter único, lineal y perfecto.

Por último, las estrategias propuestas por los docentes para superar los problemas que afectaron la concreción efectiva de planes de mejoramiento escolar se corresponden con los postulados teóricos entregados por el desarrollo organizacional escolar y por aquellas teorías sobre cambio escolar y el movimiento de la mejora de la eficacia escolar desarrollados nacional e internacionalmente. Tales medidas apuntan a la mejora de las relaciones entre los individuos y también a la generación de mayor participación de la comunidad en los procesos de cambio efectuados por el establecimiento.

Sin embargo, estas medidas sugeridas por el profesorado, desde su apreciación, son insuficientes, porque las estrategias para mejorar la implementación de planes de mejoramiento deberían llevarse a cabo en las entidades que regulan y supervisan dichos planes, es decir, en instituciones tales como el MINEDUC y la COMUDEF. Tal apreciación se corresponde con la necesidad de una redefinición de las instituciones políticas y sus roles durante procesos de cambio, entregada tanto por Bellei, Valenzuela, Vanni y Contreras (2014) y Murillo (2009).

\section{Conclusiones}

Las condiciones en las cuales se llevó a cabo la implementación de los planes de mejoramiento en el centro estudiado generaron un desgaste en el cuerpo docente y la prevalencia de bajas expectativas en cuanto a cambios que pudiese desarrollar el establecimiento a futuro.

Las condiciones desfavorables identificadas propiciaron instancias no ideales de participación para los docentes en los planes de mejoramiento escolar. Por lo tanto, la participación no comprometida que tuvo lugar durante estos procesos se constituyó como un aspecto que impidió el desarrollo de tales cambios escolares, ya 
que la conjunción de las condiciones propias del establecimiento y aquellas externas, por ejemplo, el caso de las relaciones con la COMUDEF, propició que los profesores efectuaran prácticas no idóneas para la realización de los planes de mejoramiento que debe desarrollar un establecimiento beneficiario de la Ley SEP.

Acerca de la experiencia de los docentes y las condiciones que enfrentaron durante la construcción y ejecución de los planes de mejoramiento escolar 2013-2014, fue posible observar que, pese a que el proyecto desarrollado en 2013 se caracterizó por ser más inclusivo y claro para los docentes, en contraste al implementado el 2014, la ejecución de las acciones consignadas por el establecimiento en el plan de mejora se vieron fuertemente limitadas por la imposición de acciones por parte del sostenedor COMUDEF.

Mientras que para el caso del plan de mejoramiento escolar 2014, si bien se reconoce la presencia de problemas asociados a las relaciones con el sostenedor, toman mayor relevancia aquellas condicionantes internas de la organización escolar que limitan el funcionamiento de los planes de mejoramiento escolar, tales como dificultades asociadas a la falta de objetivos y metas claras, la carencia de tiempos y espacios para la discusión de los planes, o bien la poca inclusión de la comunidad escolar y la carencia de un liderazgo democrático, entre otras.

Entonces, es posible señalar que en ambos periodos predominaron condiciones que limitaron la implementación de planes de mejoramiento que facilitaran la generación de cambios significativos en el establecimiento escolar. Además de la importancia de aquellas condicionantes de carácter organizacional y externas al establecimiento educacional, también adquirió relevancia el tipo de relaciones interpersonales que tuvo la comunidad escolar durante el desarrollo de estos planes.

Lo anterior influyó en que los funcionarios se desligaran tanto del compromiso que debían tener con los planes que efectuaba el liceo como también de sus tareas, las cuales debían contribuir 
en la mejora de la escuela. En este sentido, es posible comprender que, en su mayoría, las prácticas de los profesores no ayudaron a mejorar al establecimiento, puesto que realizaron actividades no congruentes con los proyectos de la escuela, lo que se vincula a su vez con la poca responsabilidad que reconocieron para sí como agentes que debían contribuir a la mejora de condiciones de la escuela y la educación que la institución debía entregar a la comunidad escolar.

A partir de lo anterior, es posible inferir que los problemas en las relaciones al interior de la comunidad escolar, la carencia de condiciones organizacionales óptimas, el funcionamiento no coordinado entre un establecimiento y su sostenedor, y la pérdida de protagonismo de los docentes en los planes de mejora escolar, no contribuyeron a la conformación y mantención de una cultura de la mejora al interior del centro escolar. Tal cultura facilita la implementación de planes de mejoramiento escolar.

La constitución de una cultura de la mejora requiere que exista una serie de condiciones mínimas. En primer lugar, condiciones organizacionales propias del centro escolar, tales como claridad de metas y objetivos, un liderazgo compartido, espacios y tiempos destinados a la reflexión, la participación comprometida de profesores, entre otras. En segundo lugar, son necesarias relaciones caracterizadas por la confianza entre los miembros de la comunidad escolar, lo cual facilite un trabajo de tipo mancomunado. En tercer lugar resulta necesario que los docentes asuman el proceso de cambio como una necesidad imperiosa. Por último, se requiere la adecuada interrelación entre el establecimiento y las instituciones externas que apoyan el cambio.

No obstante, si bien existieron condiciones que, de acuerdo con la visión de los docentes, favorecieron la implementación de planes de mejoramiento escolar, tales como los recursos adquiridos por el establecimiento, parte del cuerpo de profesores comprometidos y la infraestructura necesaria para llevar a cabo actividades pedagógicas, etc., prevalecieron aquellas condiciones 
poco favorables para la realización de un plan de mejoramiento escolar.

En este sentido, pese a las condiciones favorables que tiene el establecimiento, la desesperanza de los profesores en cuanto a la posibilidad de cambios dificultó la ejecución de estos. Por lo tanto, para los cambios que implemente la organización escolar a futuro es necesario mejorar las condiciones ya mencionadas, además de intentar superar aquellas bajas expectativas del profesorado en cuanto a cambios, las cuales se originan en experiencias negativas vivenciadas durante procesos realizados en años anteriores.

Además, es importante señalar que aquellas condiciones no propicias para llevar a cabo un plan de mejoramiento escolar que incida en los resultados académicos de los establecimientos educacionales, se constituye como una problemática de importancia tanto para el centro educacional estudiado, como también a nivel nacional.

La importancia de las condiciones bajo las cuales se están implementando estos planes de mejoramiento escolar radica en que la Ley SEP realiza la entrega de insumos económicos que permitan mejorar la calidad de la educación entregada a sus estudiantes, lo cual se vea reflejado en el aumento de los resultados en las mediciones nacionales. Pese a ello, como se pudo observar en esta investigación, dicha finalidad no ha sido cumplida.

Sin embargo, pese a que la concreción efectiva de los planes de mejoramiento se relaciona con las condiciones internas y externas del centro educativo, y también a las prácticas de sus funcionarios, es posible cuestionar si la Ley SEP realmente se distancia de aquellas políticas educativas tradicionales de carácter vertical y homogéneo. La experiencia del profesorado en el establecimiento estudiado permite observar que la presente Ley no pudo responder a las necesidades particulares del establecimiento. 
En definitiva, a partir de las reflexiones presentadas en este artículo y de las investigaciones que trabajan temas de mejora de la eficacia escolar, es posible señalar la existencia de una problemática general de la educación en Chile, ya que pese a la entrega de insumos económicos a los establecimientos, estos no han logrado mejorar sus resultados educacionales. Lo anterior, imposibilita la superación de las desigualdades económicas, sociales y culturales, lo cual debería ser logrado mediante un servicio educacional de calidad.

Por lo tanto, la no mejora de los resultados académicos en las mediciones nacionales son responsabilidad tanto de las instituciones políticas que supervisan el cumplimiento de dichos planes de mejora, de los centros educacionales que adquieren el compromiso de mejorar sus condiciones y eficacia escolar, como también de los docentes y miembros que conforman la comunidad educativa, quienes deben asumir tales procesos como propios e intentar constituirse como una fuerza que contribuya a los cambios del establecimiento.

Estas reflexiones permiten plantear la interrogante sobre las formas en que se administran y gestionan los recursos SEP por parte de entidades municipales y gubernamentales, y las relaciones que establecen con organizaciones escolares vulnerables durante procesos de mejora. Es decir, los procesos de planificación declarados por dichas organizaciones y su concreción en la realidad social y educativa chilena.

\section{Referencias}

Astudillo, O. (1997). Áreas organizacionales problemáticas de la escuela. En G. Undurraga, E. Astudillo, O. Astudillo, M. Manterola, y M. Pereira. Monografías en Educación. Desarrollo Organizacional Escolar (pp. 33-52). Santiago de Chile: Pontificia Universidad Católica de Chile.

Bellei, C., Valenzuela, J., Vanni, X. y Contreras, D. (2014). Lo aprendí en la escuela. ¿Cómo se logran procesos de mejoramiento escolar? Santiago de Chile: UNICEF. 
Berger, P. y Luckmann, T. (2003). La construcción social de la realidad. Buenos Aires: Amorrortu.

Bolívar, A. (2005). ¿Dónde situar los esfuerzos de mejora?: política educativa, escuela y aula. Educación y Sociedad, 26(92), 859-888.

COMUDEF (2013). Bases concurso público director/a establecimiento municipal Liceo Alto Cordillera de La Florida. Recuperado en enero de 2016 de: www.directoresparachile.cl/Repositorio/ PDFconcursos/dee_31432-3.pdf?director-establecimientomunicipal-liceo-alto-cordillera-de-la-florida

Crozier, M. y Friedberg, E. (1990). El actor y el sistema: las restricciones de la acción colectiva. México: Alianza.

Etzioni, A. (1993). Organizaciones Modernas. México: Edit. Limusa.

Fundación Chile (2010). Planes de Mejoramiento SEP: Sistematización, análisis y aprendizajes de política. Recuperado en mayo de 2016 de: http:/ / www.facso.uchile.cl/psicologia/epe/_documentos/GT_cultura_escolar_politica_educativa/recursos $\% 20$ bibliograficos/articulos $\% 20$ sep/puc(2010)estudiodiagnosticosyplanesdemejoramientosep.pdf

Hernández, V., y Santana, P. (2010). Procesos Implicados en la Mejora Escolar: Las Condiciones Institucionales. Revista Iberoamericana sobre Calidad, Eficacia y Cambio en Educación, $8(5), 45-58$

Krieger, M. (2001). Sociología de las organizaciones. Buenos Aires: Prentice Hall.

Laso, M., Albarrán, M. y Carrasco, N. (2012). Gestión Educativa Municipal: Desafíos para una Educación de Calidad en Chile. Revista iberoamericana de estudios municipales, 3(5), 95-122.

Merton, R. (2002). Teoría y estructura sociales. México: Fondo de Cultura Económica.

MINEDUC (2008). Ley de Subvención Escolar Preferencial: Un camino hacia una mayor equidad. Nuestros temas, 29, 1-12.

Murillo, F. (2009). Un marco comprensivo de mejora de la eficacia escolar. Revista Mexicana de Investigación Educativa, 9(21), 319359.

Murillo, F. y Krichesky, J. (2012). El proceso del cambio escolar: una guía para impulsar y sostener la mejora de las escuelas. Revista Iberoamericana sobre Calidad, Eficacia y Cambio en Educación, $10(1), 26-43$. 
Paredes, J. (2004). Cultura escolar y resistencias al cambio. Tendencias pedagógicas, 9, 131-142.

Pérez, G. (2007). Investigación Cualitativa. Retos e Interrogantes: II. Técnicas $y$ análisis de datos. Madrid: La Muralla.

PNUD (2009). Desarrollo humano en Chile: La manera de hacer las cosas. Santiago de Chile: Programa de las Naciones Unidas para el Desarrollo.

Raczynski, D., Muñoz, G., Weinstein, J. y Pascual, J. (2013). Subvención Escolar Preferencial (SEP) en Chile: un intento por equilibrar la macro y micro política escolar. Revista Iberoamericana sobre Calidad, Eficacia y Cambio en Educación, 11(2), 165-193.

Sepúlveda, C. y Murillo, F. (2012). El origen de los procesos de mejora de la escuela. Un estudio cualitativo en 5 escuelas chilenas. Revista Iberoamericana sobre Calidad, Eficacia y Cambio en Educación, 10(3), 7-24.

Schütz, A. (1993). La construcción significativa del mundo social. Introducción a la sociología comprensiva. Barcelona: Paidós.

Tedesco, J. (2012). Educación y Justicia Social en América Latina. Buenos Aires: Fondo de Cultura Económica/Universidad de San Martín.

UNICEF (2004). ¿Quién dijo que no se puede? Escuelas efectivas en sectores de pobreza. Santiago de Chile: Ministerio de educaciónUnicef. 